Purdue University

Purdue e-Pubs

2006

\title{
Characterization of Rough Engineering Surfaces for Use in Thermal Contact Conductance Modeling
}

\author{
A. F. Black \\ S V. Garimella \\ Purdue University, sureshg@purdue.edu
}

Follow this and additional works at: http://docs.lib.purdue.edu/coolingpubs

Black, A. F. and Garimella, S V., "Characterization of Rough Engineering Surfaces for Use in Thermal Contact Conductance Modeling" (2006). CTRC Research Publications. Paper 284.

http://dx.doi.org/10.2514/1.15519

This document has been made available through Purdue e-Pubs, a service of the Purdue University Libraries. Please contact epubs@purdue.edu for additional information. 


\title{
Characterization of Rough Engineering Surfaces for Use in Thermal Contact Conductance Modeling*
}

\author{
Anthony F. Black and Suresh V. Garimella ${ }^{\ddagger}$ \\ Cooling Technologies Research Center \\ School of Mechanical Engineering, Purdue University \\ West Lafayette, Indiana 47907-2088
}

\begin{abstract}
Most surface properties used in the calculation of contact conductance are not intrinsic to the surface, but vary with the sampling frequency of the instrument used to characterize the surface. This paper offers a methodology for characterizing a surface based on intrinsic surface characterization properties (the self-affine fractal dimension and topothesy), intrinsic material properties, and applied load. A surface characterization model is developed to predict the wavelengths on a surface that are of significance in the prediction of thermal contact conductance. The surface characterization model is combined with surface deformation and constriction resistance models to predict contact conductance across nominally flat, metallic surfaces. The long-wavelength cutoff in the surface characterization is set by the dimensions of the contact area. A theoretical correlation for the short-wavelength cutoff as a function of surface and material properties and load is developed, and then improved by a least-squares fit to experimental data. The integrated model developed predicts contact conductance in three modules: defining the unique asperity geometries important in the deformation modeling; calculating the mode of deformation of asperities; and taking into account the actual geometry of asperities in the constriction resistance model. The predicted contact conductance compares well to experimental data over a range of surface roughnesses, pressures, and substrate materials.
\end{abstract}

\footnotetext{
* Submitted for publication in AIAA Journal of Thermophysics and Heat Transfer, January 2005, and in revised form, August 2005

‡ To whom correspondence should be addressed: 765-494-5621, 765-494-0539 (FAX), sureshg@purdue.edu
} 


\section{Nomenclature}

\begin{tabular}{|c|c|}
\hline$a$ & radius of contact of an asperity \\
\hline$B$ & intercept of PSDF on a log-log plot \\
\hline$D$ & fractal dimension \\
\hline$E$ & elastic modulus \\
\hline$G$ & normalized power spectral density function (PSDF) \\
\hline$H$ & thermal contact conductance \\
\hline$I$ & length of profile scan \\
\hline$i$ & slope of structure function on a log-log plot \\
\hline$j$ & $y$-intercept of structure function on a log-log plot \\
\hline$k$ & thermal conductivity \\
\hline$L$ & applied load at interface \\
\hline$m$ & moment of the PSDF \\
\hline$N$ & number of data points in a profile scan \\
\hline$P$ & Pressure \\
\hline$Q$ & heat flow through a contact interface \\
\hline$R$ & radius of curvature and thermal resistance \\
\hline$S$ & structure function \\
\hline$T$ & Temperature \\
\hline $\mathrm{V}$ & Velocity \\
\hline$x$ & $\mathrm{x}$ (in surface plane) direction \\
\hline$Y$ & yield strength \\
\hline$z$ & profile height and axial direction \\
\hline \multicolumn{2}{|c|}{ Greek symbols } \\
\hline$\beta$ & slope of PSDF on a log-log plot \\
\hline$\delta$ & Deformation \\
\hline$\varepsilon$ & Tolerance \\
\hline$\Gamma$ & gamma function \\
\hline$\kappa$ & curvature of an asperity \\
\hline$\Lambda$ & Topothesy \\
\hline$\lambda$ & Wavelength \\
\hline$\omega$ & Frequency \\
\hline$\tau$ & horizontal separation between profile heights \\
\hline$\sigma$ & surface roughness \\
\hline \multicolumn{2}{|c|}{ Subscripts } \\
\hline$h$ & long wavelength \\
\hline ideal & objective wavelength value \\
\hline$l$ & short wavelength \\
\hline$m$ & mean value \\
\hline$n$ & order of the moment of the PSDF $n=1,2,3,4 \ldots$ \\
\hline
\end{tabular}




\section{Introduction}

Thermal contact resistance is a pervasive problem in the design of microelectronics, avionics, and space thermal management systems [1, 2]. Examples of such electronic systems include RF transistors, power electronics components, and computer chips. Contact conductance is also a problem that needs to be addressed when considering high heat flux applications such as nuclear reactors. Accurate determination of thermal contact resistance (TCR), and its inverse, thermal contact conductance (TCC), is essential to the reliable design of practically any application involving heat transfer.

Whenever two surfaces come into contact, a resistance to heat flow exists at the interface. This resistance is a result of the small fraction (usually around 1-2\% [3]) of the nominal area that is actually in contact at any given interface. Thermal contact conductance prediction may be sub-divided into the solution of three problems. The first problem is that of determining the appropriate wavelengths of the surface features involved in the physics of the contacting surfaces. The second consists of determining the extent of the deformation of those features, and the third is of determining the resistance to heat flow through those features. The present work develops a model for the first of these problems, namely, surface characterization. This surface characterization model is then combined with a surface deformation model [4] and a constriction resistance model [5] to predict the contact conductance across any nominally flat, rough, metallic interface.

Most traditional surface properties used in the calculation of contact conductance are not intrinsic to the surface, but vary with the sampling frequency of the instrument used to characterize the surface. As the resolution of the surface scanning instrument increases, finer details of the surface are captured. Figure 1 shows a typical scan of a $10 \mu \mathrm{m}(\mathrm{Ra})$ aluminum surface with various common features. It is not hard to identify that feature 1 is a peak, while 
feature 2 is not, but it is arguable whether to classify feature 3 as a peak. Similarly, feature 1 could be classified as one peak or as three (1a, 1b, 1c), and feature 4 as one peak or two. Also, if the resolution of the scanning instrument were $\tau_{2}$ instead of $\tau_{1}$, features $3,1 \mathrm{a}, 1 \mathrm{~b}$, and $1 \mathrm{c}$ would not even appear explicitly in the surface profile. For a scanning resolution of $\tau_{1}$ many more features with small radius of curvature and steep slope would appear than for a resolution of $\tau_{2}$. This creates a problem where the usual properties used to characterize a surface, such as asperity density, mean slope, and mean curvature, are not unique to a surface, but are wholly dependent on the scanning resolution of the instrument used to obtain these parameters as well as on how a peak or asperity is defined. Traditionally this problem has been addressed in contact conductance by requiring a statement of the sampling frequency of the instrument used to obtain predicted values, though this guideline is not often followed. Ideally, the surface should be characterized by parameters that remain constant, independent of sampling frequency, if such intrinsic properties can be found.

\section{Intrinsic Surface Characterization}

Because surface roughness is such an important factor in so many scientific fields, many researchers have developed surface parameters devised to characterize a surface for their particular application. Some of the more common properties used are bearing ratio, asperity density, center line average surface roughness, and the already mentioned RMS peak curvature and asperity slope. With the exception of surface roughness, all of these parameters are dependent on the sampling frequency.

All surface deformation and contact conductance models, whether statistical or deterministic, are dependent on the properties of the surface such as mean asperity slope, radius of curvature, and contact spot density. A recent contact conductance model by Milanez et al. [6] 
accounts for the fact that most engineering surfaces do not exhibit a truly Gaussian distribution of asperity heights; a truncated Gaussian scheme was presented to predict deformation and contact conductance based on mean asperity slope and contact spot density. Thomas [7] showed that these properties are not unique for a given surface, but change with the sampling rate of the instrument used to characterize the surface. Ju and Farris [8] also demonstrated the dependence of RMS curvature and slope on the sampling frequency. They showed that the RMS curvature can vary by more than three orders of magnitude and the RMS slope can vary by almost two orders of magnitude while the RMS height stays essentially constant as the sampling interval goes from 0.1 to $0.0001 \mathrm{~mm}$. The properties of slope and radius of curvature are particularly sensitive to the sampling rate at which the surface is characterized. Whitehouse and Archard [9] showed that for a random surface with a Gaussian distribution of surface heights, the slope increases as the inverse of the square root of the sampling interval, and the peak radius of curvature decreases proportionally as the $3 / 2$ power of the sampling interval.

In order to obtain surface deformation and constriction resistance results that are independent of the resolution of the scanning instrument, long-wavelength and short-wavelength cutoffs must be defined. The appropriate long-wavelength for the case of contact conductance is determined from the area of contact. On the other hand, the short-wavelength cutoff is not so easily discernible. As a further confounding factor, it is likely that the critical short wavelength is not a constant for a given surface but will increase under increasing load because larger asperities will deform plastically under ever-increasing loads. The present work seeks a shortwavelength cutoff criterion that is independent of the mode of deformation, but depends only on the surface properties, material properties, and load. 


\section{$\underline{\text { Intrinsic Surface Properties }}$}

Several researchers $[10,11,12]$ have shown that most engineering surfaces exhibit fractal behavior over at least part of their range of surface wavelengths. Sayles and Thomas [10] plotted the normalized power spectral density function of several different engineering surfaces as a function of wavelength and obtained a straight line for all of the surfaces used. The fact that all of these surfaces lie on a straight line when plotted on a log-log scale indicates that they are of a self-affine fractal nature and may be described by the equation,

$$
G(\omega)=B \cdot \omega^{\beta}
$$

where $B$ has dimensions of length and $\beta$ is a nondimensional constant from which the fractal dimension, $D$, may be determined. Since B and $\beta$ are constant over a wide range of wavelengths, they are intrinsic properties of the surface. Nayak [13] used statistical theories to predict surface parameters and showed that asperity density and curvatures may be defined as the second $\left(m_{2}\right)$ and fourth $\left(m_{4}\right)$ moments of the power spectral density function (PSDF), $G(\omega)$, of a Gaussian surface, where:

$$
m_{n}=\int_{\omega_{1}}^{\omega_{2}} \omega^{n} G(\omega) d \omega
$$

and $\omega_{1}$ and $\omega_{2}$ are the low- and high-frequency cutoffs, respectively, and are translated to the corresponding long- $\left(\lambda_{h}\right)$ and short-wavelengths $\left(\lambda_{l}\right)$ using $\omega=2 \pi / \lambda$. Majumdar and Bhushan [14] attempted to circumvent the problem of defining a short-wavelength cutoff by predicting contact behavior from contact size alone. Majumdar and Tien [12] used this approach to model contact conductance. Rosén et al. [15] pointed out that the results in [14] are counter-intuitive and that the theory is not satisfactory because it does not take into account the effect of asperity geometry in the deformation model and thus, in the contact conductance prediction as well. Thomas and Rosén [16] presented an argument for a short-wavelength cutoff based on the 
plasticity index of Greenwood and Williamson [17]. Their argument assumes that any asperities smaller than a given wavelength will deform plastically under the lightest loads and hence will not be important in the deformation mechanics of a surface.

\section{Fractal Dimension and Topothesy of a Profile Scan}

In order to avoid the difficulties involved in calculating the power spectral density function, the method proposed by Sayles and Thomas [10] is adopted. This method uses the structure function to compute the fractal dimension, $D$, and a surface property denoted in [10] as the topothesy, $\Lambda$ :

$$
S(\tau)=\frac{1}{I-\tau} \int_{0}^{I-\tau}\{z(x)-z(x+\tau)\}^{2} d x
$$

Here, $\tau$ is the separation between two profile heights, $z(x)$, and $I$ is the length of the profile. The physical meaning of the fractal dimension and topothesy are described in the sub-section below; additional details may be found in $[12,16]$. Russ [18] showed that the structure function of a fractal profile also obeys a power law and may be plotted as a straight line on a log-log scale as shown in Figure 2. The fractal dimension and topothesy were also determined to be related to the structure function as:

$$
S(\tau)=\Lambda^{2 D-2} \tau^{2(2-D)}
$$

Once the structure function of a profile is computed the fractal dimension and topothesy may be obtained from the slope, $i$, and y-intercept, $j$, of the linear portions of the structure function plot on a log-log scale as follows:

$$
\begin{gathered}
i=2(2-D) \\
j=(2 D-2) \log (\Lambda)
\end{gathered}
$$


Figure 2 shows that no surface has the same fractal dimension over all wavelengths, and so, there is a change in slope at a particular frequency. Majumdar and Tien [12] referred to this as the corner frequency and showed that the change in the nature of the surface at this frequency is due to a change in the mode of preparation of the surface.

The range of wavelengths of interest for the contacting asperities at light loads is in the low-wavelength region of the structure function (i.e., $\log (\tau)<2$ in Figure 2). This was verified by a trial and error method as described below. Hence the low-wavelength region of the structure function is used to calculate the fractal dimension and topothesy. The slope and yintercept of this region are found by a least-squares fit of a straight line through the lowest few points of the structure function. Since the number of data points used in calculating the structure function is not known beforehand, either three, or the total number of data points divided by 1000 (whichever is greater), data points were used for the straight-line fit. One thousand was chosen as a number that appears to give a sufficient number of data points below the corner frequency to yield a good curve-fit. The parameters $D$ and $\Lambda$ are then found by using Equations (5) and (6).

\section{Physical Meaning of Fractal Dimension and Topothesy}

Fractal geometry is used to describe shapes where the same basic structural pattern appears upon increasing magnification of the object. The fractal dimension is best illustrated by the three cases shown in Figure 3 and Figure 4. In Figure 3 the basic structure of the line can be divided into self-similar lines of length $1 / m$. The total length is independent of $1 / m$ and the number of self-similar lines, $N$, is proportional to $m$. The basic structure of a square can be divided into self-similar squares of dimensions $1 / \mathrm{m}^{2}$. Magnifying the square by $m$ will show a square that is similar to the original. The number of squares making up the whole is proportional to $m^{2}$. Generally, the number of self-similar units that make up the whole is given by: 


$$
N \sim m^{D}
$$

Figure 4 shows the self-similar Koch curve of fractal dimension $D=1.26$ [12]. Self-affine structures are fractal structures where the object requires different magnifications in different directions in order to maintain the same basic structural pattern. Hence, an additional parameter is needed to describe a self-affine structure. This parameter is the topothesy, $\Lambda$, and is defined as the horizontal distance over which the slope of the surface will, on average, be one radian [19]. Majumdar and Tien [12] illustrated the effect of the fractal dimension, D, and a parameter similar to the topothesy, $\mathrm{G}$, on a generated surface profile. Reducing $\mathrm{G}$ was shown to decrease the amplitude of the surface roughness over all frequencies; increasing D caused more of the high-frequency components to become significant in the roughness.

\section{Physically Significant Surface Wavelengths for Deformation and Constriction Resistance}

Nayak [13] showed that for a Gaussian surface the curvature $(\kappa)$ and the fourth moment of the PSDF are related by:

$$
\frac{\kappa}{\sqrt{m_{4}}}=\frac{8}{3 \sqrt{\pi}}
$$

The radius of a sphere as used in the deformation model is related to the curvature by:

$$
\kappa=1 / R
$$

This gives the radius of a sphere in terms of the fourth moment of the PSDF as follows:

$$
R=\frac{3}{8} \sqrt{\frac{\pi}{m_{4}}}
$$

A need to filter is assumed based on the purely geometrical assumption that after a sphere deforms beyond its radius it no longer models the behavior of an asperity deforming in the same manner, so:

$$
\delta=R
$$


The short-wavelength criterion should be independent of mode of deformation. Since the deformation analysis of Singhal and Garimella [4] models the contacting asperities as spheres of radius $R$, it is assumed that any asperities deforming beyond the radius of the sphere (i.e., beyond $\delta=R$ ) will need to be filtered to avoid geometric inconsistencies between spheres and the asperities. A quick check of the minimum plastic deformation limit developed in [4] reveals that this assumption places the mode of deformation of such asperities in the plastic region where the radius of contact, $a$, is given by:

$$
a^{2}=2 \delta R
$$

And the mean pressure on the sphere is given by:

$$
\frac{P_{m}}{Y}=3
$$

The mean pressure on the sphere may also be assumed to be equal to the load applied divided by the area of contact which, when combined with Equations (11) and (12), gives the following:

$$
P_{m}=\frac{L}{\pi a^{2}}=\frac{L}{2 \pi R^{2}}
$$

Combining Equations (13) and (14) gives:

$$
R^{2}=\frac{L}{6 \pi Y}
$$

Now, combining Equations (10) and (15) gives the fourth moment of the PSDF as a function of the applied load and the yield strength of the material:

$$
R=\sqrt{\frac{L}{6 \pi Y}}=\frac{3}{8} \sqrt{\frac{\pi}{m_{4}}}
$$

The moments of the PSDF are given by Equation (2), and for any engineering surface that exhibits self-affine fractal behavior the PSDF is given by Equation (1). Solving for $\mathrm{m}_{4}$ in Equation (16) and substituting Equation (2) into Equation (1) with $n=4$, and assuming the highfrequency components to be much larger than the low-frequency components gives: 


$$
m_{4}=\frac{54 \pi^{2} Y}{64 L}=\frac{B}{\beta+5} \omega_{l}^{\beta+5}
$$

Russ [18] showed that the parameters of the PSDF, $B$ and $\beta$, are related to the fractal dimension $(D)$ and the topothesy $(\Lambda)$ by the equations:

$$
B=\frac{(2-D) \Lambda^{2 D-2}}{\sin \left\{\pi\left(\frac{2 D-3}{2}\right)\right\} \Gamma(2 D-3)}
$$

and

$$
\beta=2 D-5
$$

In order to avoid calculating the Gamma Function of a negative number, Equation (18) was converted to the form:

$$
B=\frac{(2-D)(2 D-3) \Lambda^{2 D-2}}{\sin \left\{\pi\left(\frac{2 D-3}{2}\right)\right\} \Gamma(2 D-2)}
$$

The wavelength is determined from the frequency by:

$$
\omega=\frac{2 \pi}{\lambda}
$$

Substituting Equations (21), (20), and (19) into Equation (17), gives:

$$
\frac{54 \pi^{2} Y}{64 L}=\frac{(2-D)(2 D-3)\left(2 \pi / \lambda_{1}\right)^{2 D} \Lambda^{2 D-2}}{2 D \sin \left\{\pi\left(\frac{2 D-3}{2}\right)\right\} \Gamma(2 D-2)}
$$

Finally, solving for $\lambda_{1}$, rearranging and non-dimensionalizing, the final form of the solution is obtained:

$$
\left(\frac{\lambda_{l}}{\Lambda}\right)^{2 D}=\frac{64 L(2-D)(2 D-3)(2 \pi)^{2 D}}{108 \pi^{2} D \sin \left\{\pi\left(\frac{2 D-3}{2}\right)\right\} \Gamma(2 D-2)}\left(\frac{L}{Y \Lambda^{2}}\right)
$$


The predicted short-wavelength cutoff is nondimensionalized with the topothesy and plotted in Figure 5 versus the nondimensional group $\mathrm{L} / \mathrm{Y} \Lambda^{2}$. Figure 6 shows the same information with an objective short-wavelength cutoff, $\lambda_{\text {ideal}}$, which was found by a trial-anderror method of guessing a short-wavelength cutoff, running the predictive code for thermal contact conductance, and adjusting the cutoff until the predicted contact conductance matched the experimental data from Singhal et al. [20]. A first-order Butterworth filter was used to filter the surface data to the desired frequency. Comparing Figure 5 with Figure 6, the predicted values of the short-wavelength cutoff are seen to be an order of magnitude too high (the two figures have different scales). When applied to the surface, filtering to these wavelengths causes a severe under-prediction of the contact conductance. Due to the removal of so many of the short wavelengths and the flattening of the peak heights that are important in the deformation mechanics of the surface, the filtered surface no longer resembles the original surface from which it is derived.

Figure 6 shows that, while there is a lot of scatter in the objective cutoff wavelengths, the general trend of the predicted wavelength shown in Figure 5 applies to the trial-and-error objective data. Therefore, to predict the correct cutoff wavelengths for a surface, an equation of the general form of Equation (23) was fit to the data of Figure 6. A least-squares fit to the data yields:

$$
\left(\frac{\lambda_{l}}{\Lambda}\right)^{2 D}=4.043 \times 10^{-6} \frac{64(2-D)(2 D-3)(2 \pi)^{2 D}}{108 \pi^{2} D \sin \left\{\pi\left(\frac{2 D-3}{2}\right)\right\} \Gamma(2 D-2)}\left(\frac{L}{Y \Lambda^{2}}\right)^{1.192}
$$

The average error in the short-wavelength cutoff from the objective cutoff is $43.6 \%$ with a maximum of $196 \%$ (for the $1 \mu \mathrm{m}$ brass surface) and a minimum of $0.15 \%$ (for the $5 \mu \mathrm{m}$ aluminum surface). At first glance the error in the equation seems substantial, but the wide scatter in the objective values may be ameliorated by a more robust filtering method as explained 
below. In any case, the predicted contact conductance values do not suffer substantially from this error.

\section{Results and Discussion}

The process of filtering a profile scan using a first-order Butterworth filter eliminates the undesired surface wavelengths, but it also has an undesired smoothing effect on the asperity heights. The peaks that are predicted to be in contact at a given load change due to the reduction of asperity height caused by filtering. An asperity predicted to be in contact is shown in Figure 7. It can be seen that the unfiltered surface would over-predict the contact area because it predicts that features $2 \mathrm{a}$ and $2 \mathrm{~b}$ are both in contact, whereas one asperity would better represent the actual contact at the given deformation. Additionally the reduction in peak height due to the filtering causes a change in the predicted contact area or even, as in the case of feature 1 at a slightly higher load, whether or not an asperity is in contact at a given load.

Another significant effect of filtering the surface is shown in Figure 8. As the surface gets filtered to increasing short-wavelength cutoffs, the two asperities shown begin to merge into one asperity. Just before they merge, their radii of curvature increase at least tenfold. This substantial increase in the radius of curvature causes the mean plane separation at that particular load to increase substantially, reducing the predicted area of contact and hence the contact conductance (Figure 9). After these asperities merge, the radius of curvature returns to a wellbehaved value, as does the contact conductance prediction. Ideally, the filtering should remove the undesired surface wavelengths while more or less maintaining the peak heights. This may be accomplished by using a different-order Butterworth filter, a different filter altogether, or perhaps even by using wavelet analysis. Additionally there may be some features that do not need filtering, and so a local filtering method could be used in conjunction with one of these 
filters so as to selectively filter only those asperities that need filtering. For example, feature 2 in Figure 7 would be filtered to remove the small asperities (features 2a and 2b) while leaving feature 1 unmodified because it does not have any smaller features that need to be filtered. This type of scheme would give a more accurate picture of asperity deformation under a given load, and hence reduce the variability in the objective wavelength data and improve the predicted values of contact conductance by decreasing the error in the fitted equation. In the present work the non-monotonically increasing contact conductance with load, caused by the erratic behavior of the asperity geometry with filtering, is accounted for by fitting a smooth curve to the predicted values of contact conductance.

Comparison to Experimental Results for Isotropically Rough, Bare Surface Contacts

The short-wavelength cutoffs of Equation (16) were used in the model developed in this work to predict contact conductance for the experiments of Singhal et al. [20]. Results from six different cases are compared to experimental data in Figure 10 - Figure 15. The materials used in the experiments were aluminum, brass, and copper, all with isotropically rough surfaces ranging from 1 to $15 \mu \mathrm{m}$ center-line average (CLA) roughnesses; relevant properties are listed in Table 1. The raw profile data were obtained for all surfaces with a SurfAnalyzer 5400 stylus profilometer at a sampling interval of $1.5625 \mu \mathrm{m}$. Profile data were taken from the surfaces both before (pre-loading) and after testing (post-loading). In addition to the pre-loading and postloading filtered-profile predictions, Figure 10 also shows the predicted contact conductance values obtained using the unfiltered profile scans. The fitted numerical results shown in Figure 10 represent a relation of the form $h=L^{c}$ where $c$ is a constant less than one. This follows the commonly used contact conductance relationship

$$
\frac{h \sigma}{k A}=c_{1} \sigma^{\prime}\left(\frac{L}{H A}\right)^{c_{2}}
$$


in which $h$ is the contact conductance, $\sigma$ the RMS surface height, $\sigma^{\prime}$ the RMS slope, $k$ the thermal conductivity, $L$ the load applied to the interface, $H$ the hardness of the softer material, $A$ the apparent area of contact, and $c_{1}$ and $c_{2}$ are constants [2, 21]. The fit is employed in the plots to eliminate any fluctuations in some of the predictions.

Some degree of fluctuation can arise in the predictions because the surface characterization and the deformation calculations have different effects on the predicted contact conductance. Increasing the load in the deformation model tends to increase the contact conductance as would be expected, but it also increases the short-wavelength cutoff and filters out some of the smaller wavelengths. The larger short-wavelength cutoff tends to smooth the asperity peak heights and increase the radius of curvature of the contacting asperities. Thus, while the deformation model correctly predicts an increase in the contact conductance with load, the surface characterization model moderates this increase by ensuring that the correct size of asperities at the given load are being used in the deformation model. However, the filtering is applied to some asperities where it is not needed and changes the contact mechanics of those asperities when no changes should have been made. While this leads to minor fluctuations in the predicted contact conductance as a function of load for some cases, the effect is generally small. These fluctuations would be eliminated if a more robust method of filtering were used that maintains the asperity height while eliminating short-wavelengths as discussed above. In subsequent figures in this work, only the experimental post-load data are shown along with the fitted numerical post-load data for clarity.

Filtering the data to the predicted wavelengths from Equation (24) improves the predicted values of the contact conductance over the unfiltered predictions for all cases studied in this work. The predicted values match the experimental values very well for the $1 \mu \mathrm{m}, 5 \mu \mathrm{m}$, and 15 $\mu \mathrm{m}$ aluminum samples (Figure 10, Figure 11, and Figure 13). The $5 \mu \mathrm{m}$ brass (Figure 14) and 5 
$\mu \mathrm{m}$ copper (Figure 15) predictions also match the experimental values to within a reasonable degree of accuracy. The deviation in the predictions from the experimental values in the case of $10 \mu \mathrm{m}$ aluminum (Figure 12) are mostly due to the error between the fitted short-wavelength cutoff (Equation 24) and the objective short-wavelength cutoff.

Also of interest is the change in slope of the experimental TCC values at different loads, most clearly visible in Figure 15. This may be due in part to the nature of the surface. As the surface begins to deform under extremely light loads, the smallest asperities will assume the greatest importance. As the load increases to moderately light loads these smallest asperities combine to form larger asperities which are harder to deform and hence a leveling off of the percent increase in contact area (and hence TCC) is seen for a given increase in load.

\section{Conclusions}

A surface characterization model is developed to properly characterize a surface for prediction of surface deformation and thermal contact conductance under light loads. Most surface characterization properties (RMS radius of curvature, slope, bearing ratio, etc.) are not intrinsic properties of the surface, but vary with resolution of the scanning instrument. Therefore, a method for characterizing a surface based on intrinsic surface properties (fractal dimension and topothesy), the load applied to the surface, and the yield strength of the material is developed. The surface characterization model determines the shortest wavelengths critical to the physics involved in the deformation of asperities at a given load. The model yields predictions for TCC that are close to experimental values as a function of load for several different surfaces and materials.

The predictive tool developed for thermal contact conductance is limited to a consideration of nominally flat, metallic surfaces. Also, the deformation model of Singhal and 
Garimella [4] used in this work is valid only for metals and so a different deformation model needs to be developed for non-metallic surfaces. The surface characterization model should be improved to include a local filtering process that removes small features deforming beyond their radius of curvature while leaving the other features unmodified. A higher-order filter, or different method of removing unwanted wavelengths, should be used to reduce the smoothing effect caused by the filter used here.

\section{References}

1 Yovanovich, M.M., and Antonetti, V.W., 1988, "Application of Thermal Contact Resistance Theory to Electronic Packages," Advances in Thermal Modeling of Electronic Components, Vol. 1, pp. 79-128.

2 Madhusudana, C.V., 1966, Thermal Contact Conductance, Springer-Verlag, New York.

3 Bowden, F.P., and Tabor, D., 1950, The Friction and Lubrication of Solids, Oxford University Press, London, pp. 20-32.

4 Singhal, V., and Garimella, S.V., 2001, "Prediction of Thermal Contact Conductance by Surface Deformation Analysis," in Proceedings of International Mechanical Engineering Congress and Exposition, New York, IMECE2001/HTD-24376, pp. 1-8.

5 Black, A.F., Singhal, V., and Garimella, S.V., 2004, "Analysis and Prediction of Constriction Resistance for Contact Between Rough Engineering Surfaces,” Journal of Thermophysics and Heat Transfer, Vol. 18, pp. 30-36.

6 Milanez, F. H., Yovanovich, M. M., and Culham, J. R., 2002, "Effect of Surface Asperity Truncation on Thermal Contact Conductance," ITHERM VIII, May 29 - June 1, San Diego, CA.

7 Thomas, T.R., 1999, Rough Surfaces, Imperial College Press, London.

8 Ju, Y., and Farris, T.N., 1996, "Spectral Analysis of Two-Dimensional Contact Problems," Journal of Tribology, Vol. 118, pp. 320-328.

9 Whitehouse, D.J., and Archard, J.F., 1970, “The Properties of Random Surfaces of Significance In Their Contact,” Proceedings of the Royal Society, Vol. A316, pp. 97-121.

10 Sayles, R.S., and Thomas, T.R., 1978, "Surface Topography As A Non-stationary Random Process,” Nature, Vol. 271, pp. 431-434.

11 Thomas, T.R., Rosén, B.-G., and Amini, N., 1999, "Fractal Characterisation of the Anisotropy of Rough Surfaces,” Wear, Vol. 232, pp.41-50.

12 Majumdar, A., and Tien, C.L., 1990, "Fractal Characterization and Simulation of Rough Surfaces,” Wear, Vol. 136, pp. 313-327. 
13 Nayak, P.R., 1971, “Random Process Model of Rough Surfaces,” Journal of Lubrication Technology, Vol. 93, pp. 398-407.

14 Majumdar, A., and Bhushan, B., 1990, "Fractal Model of Elastic-Plastic Contact Between Rough Surfaces,” Journal of Tribology, Vol. 113, pp. 205-216.

15 Rosén, B.-G., Ohlsson, R., and Thomas, T.R., 1998, "Nano Metrology of Cylinder Bore Wear,” International Journal of Machine Tools and Manufacture, Vol. 38, pp. 519-527.

16 Thomas, T.R., and Rosén, B.-G., 2000, "Determination of the Optimum Sampling Interval for Rough Contact Mechanics,” Tribology International, Vol. 33, pp. 601-610.

17 Greenwood, J.A., and Williamson, J.B.P., 1966, “Contact of Nominally Flat Surfaces,” Proceedings of the Royal Society, Vol. A295, pp. 300-319.

18 Russ, J.C., 1994, Fractal Surfaces, Plenum Press, New York.

19 Berry, M.V., 1979, "Difractals," Journal of Physics, Vol. A12, 781-797

20 Singhal, V., Litke, P.J., Black, A.F., and Garimella, S.V., 2005, “An Experimentally Validated Thermo-mechanical Model for the Prediction of Thermal Contact Conductance," International Journal of Heat and Mass Transfer, in press.

21 Mikic, B.B., 1974, "Thermal Contact Conductance: Theoretical Considerations,” International Journal of Heat and Mass Transfer, Vol. 17, pp. 205-214. 


\section{List of Tables}

Table 1. Properties of materials used in the experiments in [20].

\section{List of Figures}

Figure 1. Portion of a $10 \mu \mathrm{m}$ Ra aluminum profile scan taken using a SurfAnalyzer 5400 with a sampling interval of $1.5 \mu \mathrm{m}$ illustrating the importance of sampling interval $(\tau)$ on the surface properties.

Figure 2. A log-log plot of the structure function computed at various sampling rates (wc) for an aluminum surface of $5 \mu \mathrm{m}$ center line average roughness.

Figure 3. Self similarity of a line $(D=1)$ and a square $(D=2)$ (adapted from [12]).

Figure 4. Self-similar Koch curve with fractal dimension $D=1.26$ (adapted from [12]).

Figure 5: Theoretical short-wavelength cutoff as a function of load, topothesy, and yield strength of the substrate material.

Figure 6: Objective (Ideal) short-wavelength cutoff and the theoretical Equation (24) fitted to experimental data [20].

Figure 7: Unfiltered and filtered $10 \mu \mathrm{m}$ aluminum scan along with predicted deformation at $2600 \mathrm{~N}$ load.

Figure 8: Effect of filtering on two small asperities as they merge into one large asperity.

Figure 9: Predicted thermal contact conductance of a $1 \mu \mathrm{m}$ Ra aluminum surface at a load of $1550 \mathrm{~N}$ as a function of cutoff wavelength.

Figure 10: Comparison of experimental contact conductance versus load for a $1 \mu \mathrm{m}$ rough aluminum surface to numerically predicted values for filtered profile scans; predictions from unfiltered scans are also shown.

Figure 11: Comparison of experimental contact conductance versus load for a $5 \mu \mathrm{m}$ rough aluminum surface to predicted values from post-load scans.

Figure 12: Comparison of experimental contact conductance versus load for a $10 \mu \mathrm{m}$ rough aluminum surface to predicted values from post-load scans.

Figure 13: Comparison of experimental contact conductance versus load for a $15 \mu \mathrm{m}$ rough aluminum surface to predicted values from post-load scans.

Figure 14: Comparison of experimental contact conductance versus load for a $5 \mu \mathrm{m}$ rough brass surface to predicted values from post-load scans.

Figure 15: Comparison of experimental contact conductance versus load for a $5 \mu \mathrm{m}$ rough copper surface to predicted values from post-load scans. 
Table 1. Properties of materials used in the experiments in [20].

\begin{tabular}{|c|c|c|c|c|}
\hline Material & $\begin{array}{c}\text { Yield } \\
(\mathrm{MPa})\end{array}$ & $\begin{array}{c}\text { Young's Modulus } \\
(\mathrm{GPa})\end{array}$ & $\begin{array}{c}\text { Conductivity } \\
(\mathrm{W} / \mathrm{mK})\end{array}$ & $\begin{array}{c}\text { Poisson's } \\
\text { Ratio }\end{array}$ \\
\hline Aluminum & 358.07 & 69 & 184.9 & 0.33 \\
\hline Copper & 451.26 & 110 & 397.4 & 0.34 \\
\hline Brass & 573.90 & 97 & 98.7 & 0.32 \\
\hline
\end{tabular}




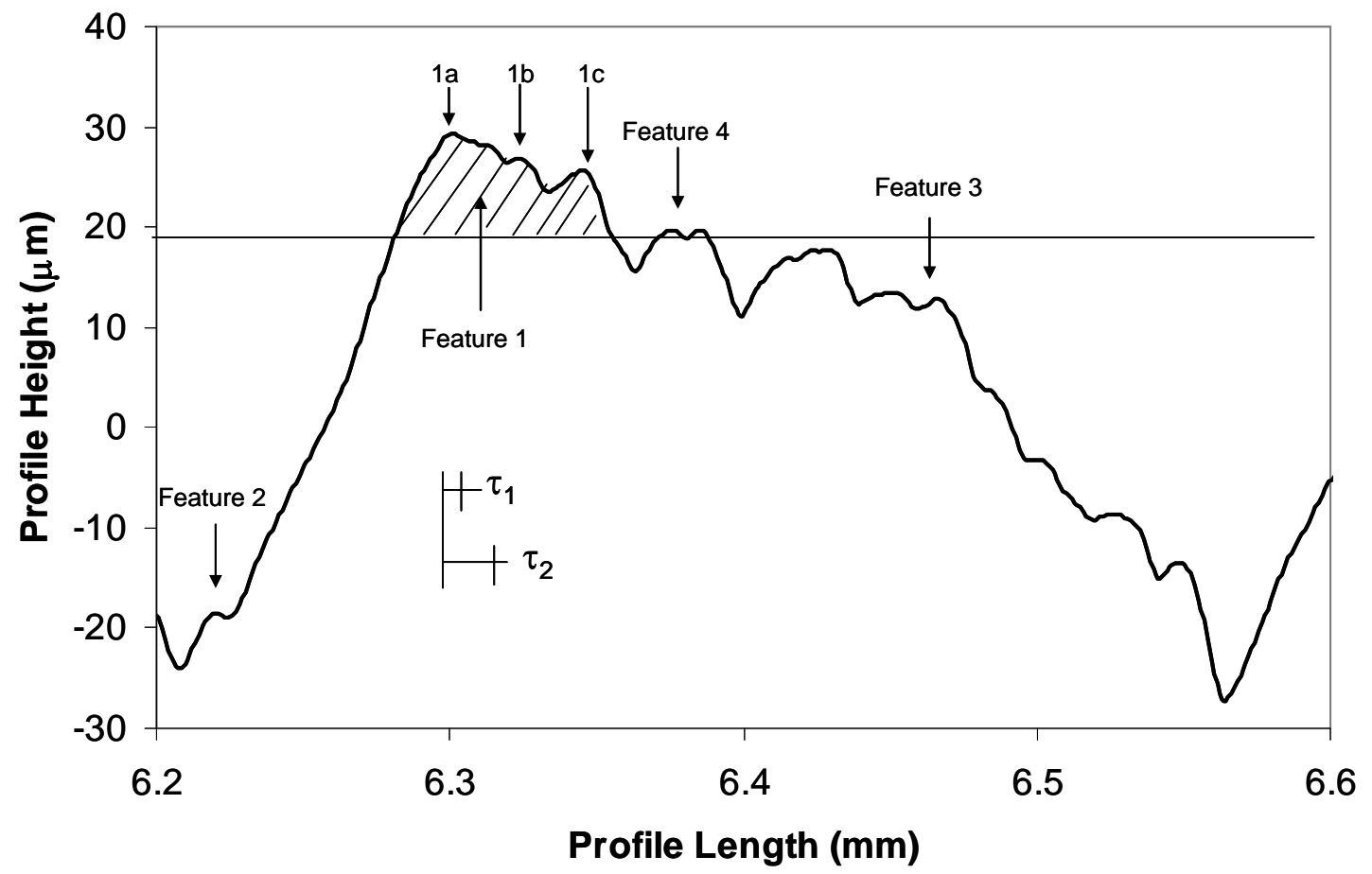

Figure 1. Portion of a $10 \mu \mathrm{m}$ Ra aluminum profile scan taken using a SurfAnalyzer 5400 with a sampling interval of $1.5 \mu \mathrm{m}$ illustrating the importance of sampling interval $(\tau)$ on the surface properties. 


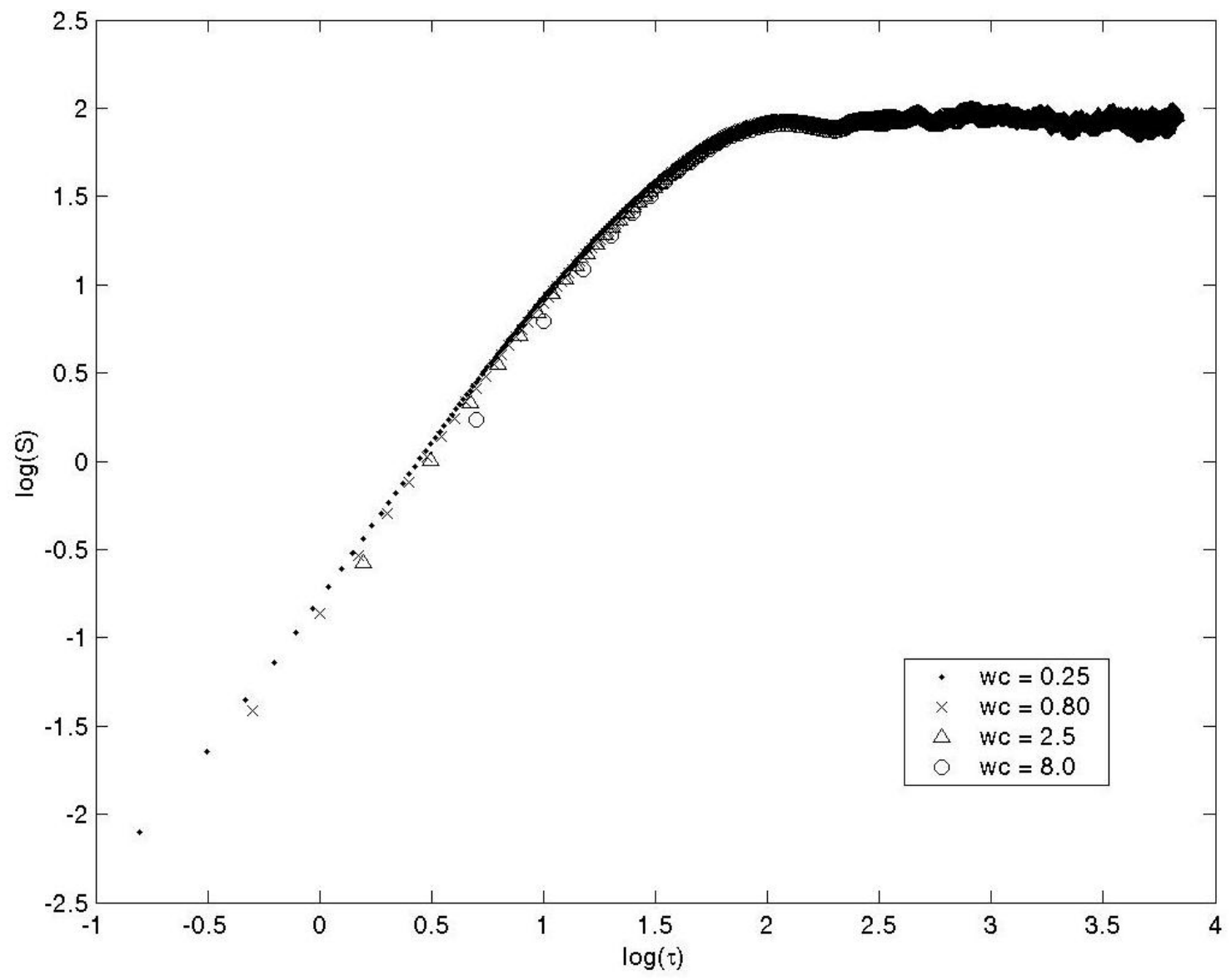

Figure 2. A log-log plot of the structure function computed at various sampling rates (wc) for an aluminum surface of $5 \mu \mathrm{m}$ center line average roughness. 

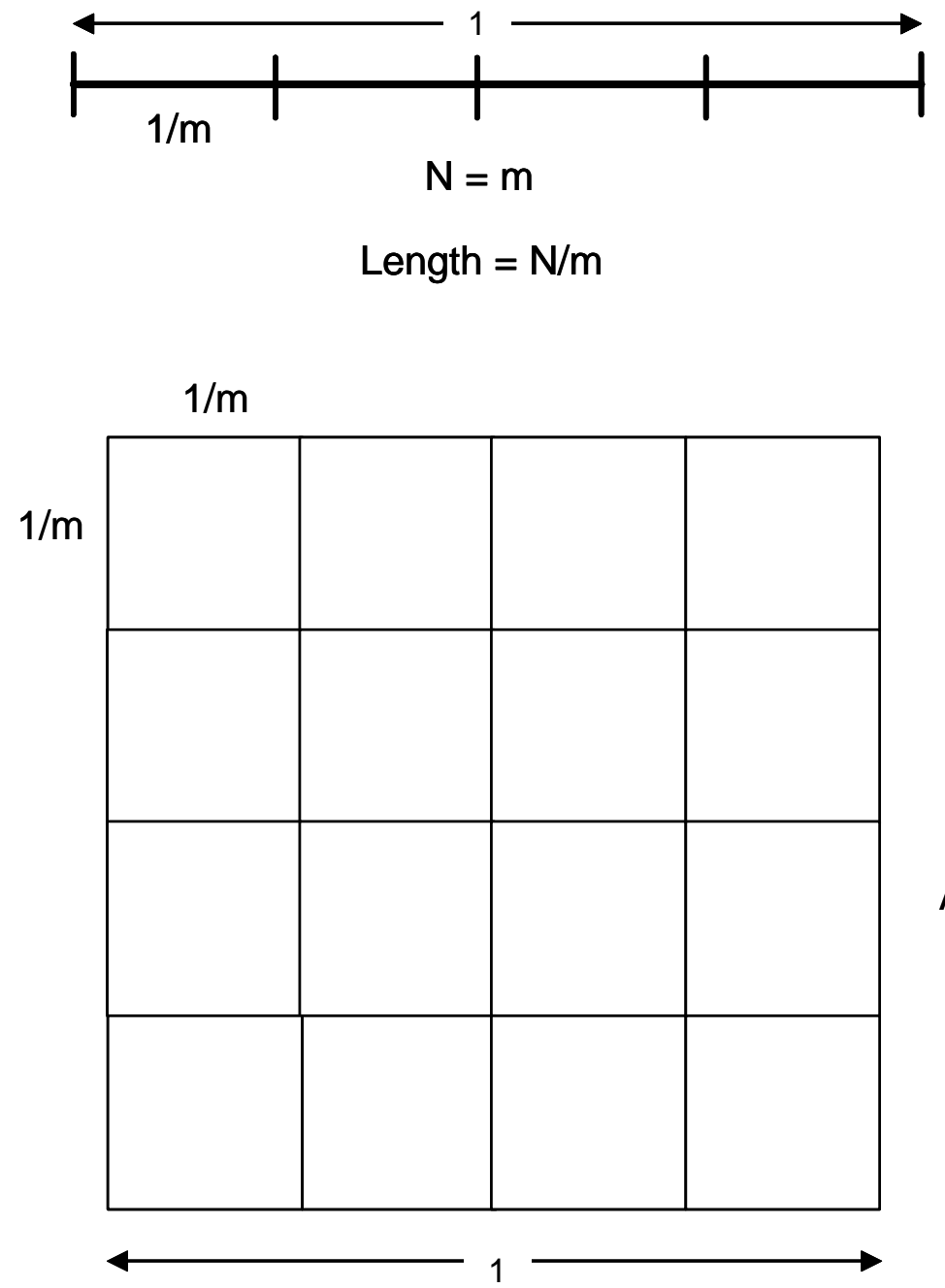

$$
\mathrm{N}=\mathrm{m}^{2}
$$

Area $=\mathrm{N} / \mathrm{m}^{2}$

Figure 3. Self similarity of a line $(D=1)$ and a square $(D=2)$ (adapted from [12]). 

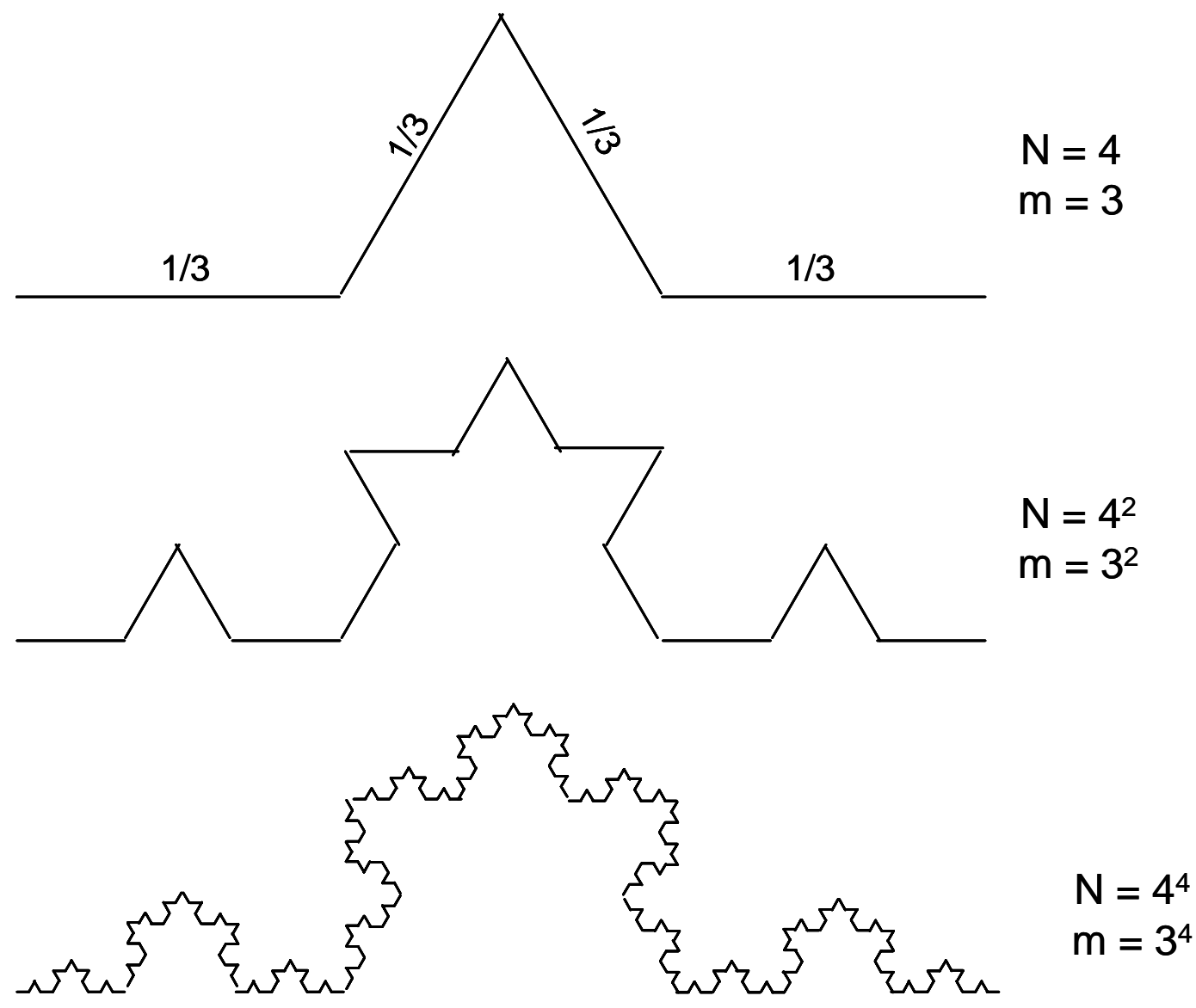

\section{$D=\log N / \log m=1.26$}

Figure 4. Self-similar Koch curve with fractal dimension D = 1.26 (adapted from [12]). 


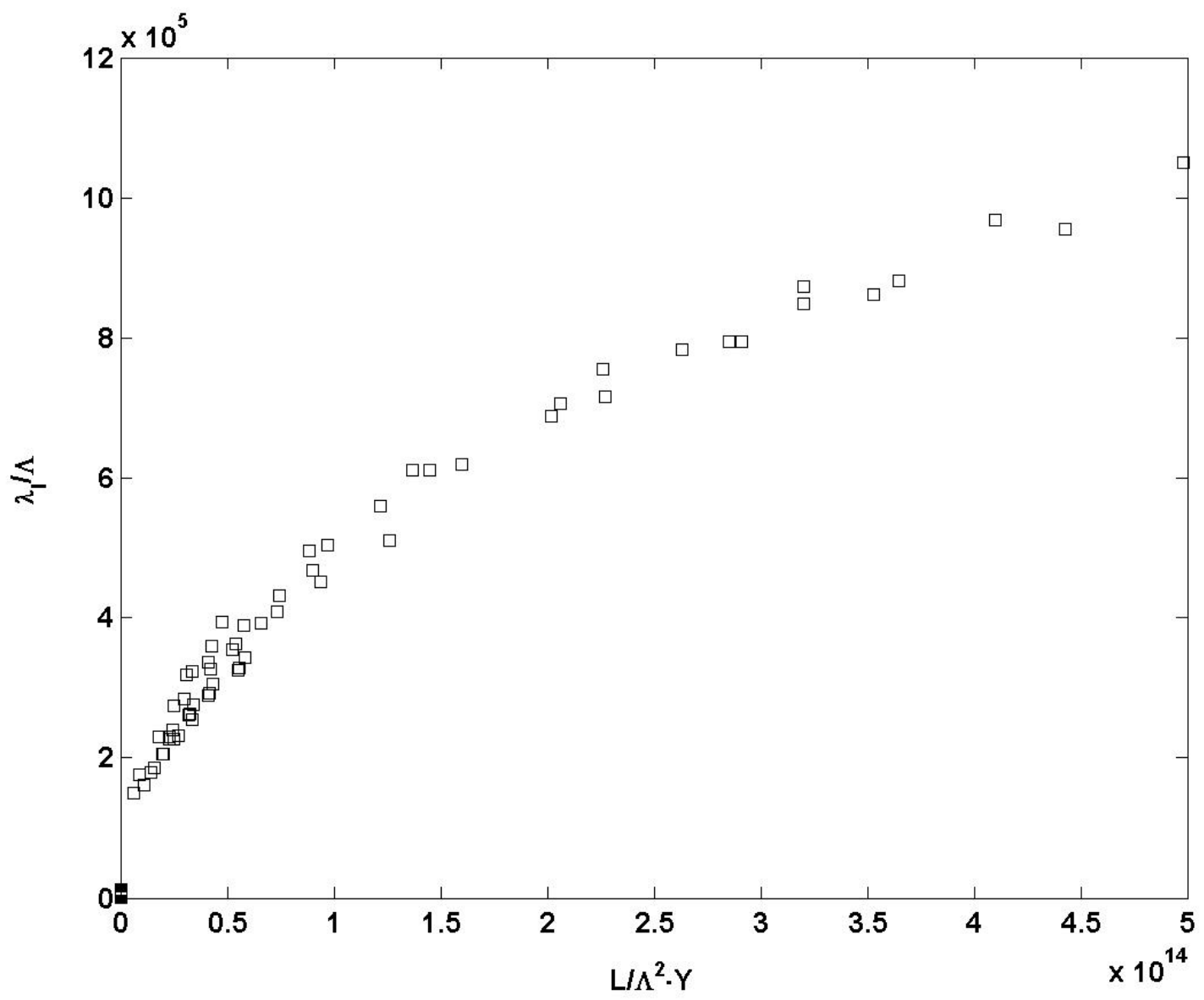

Figure 5: Theoretical short-wavelength cutoff as a function of load, topothesy, and yield strength of the substrate material. 


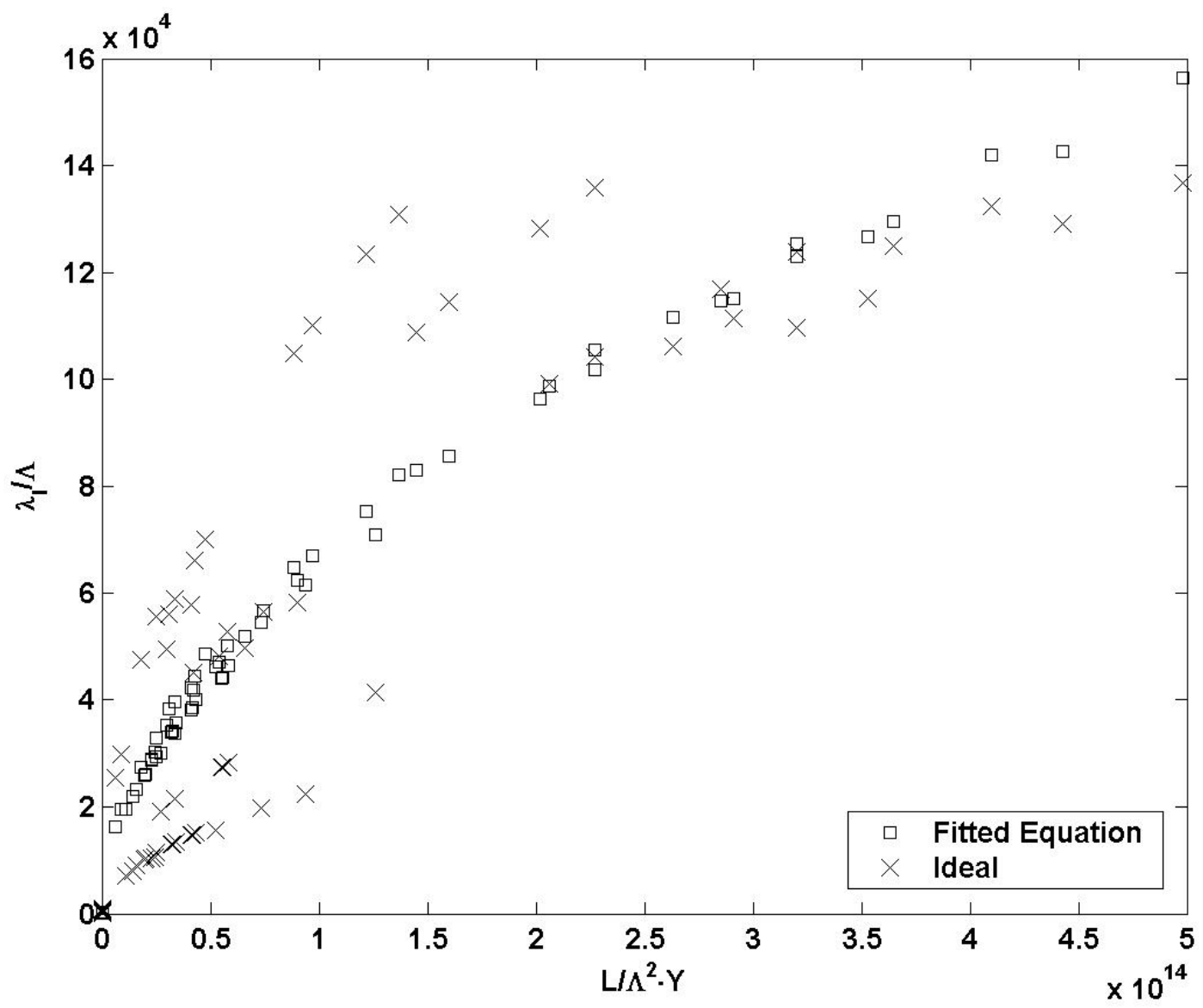

Figure 6: Objective (Ideal) short-wavelength cutoff and the theoretical Equation (24) fitted to experimental data [20]. 


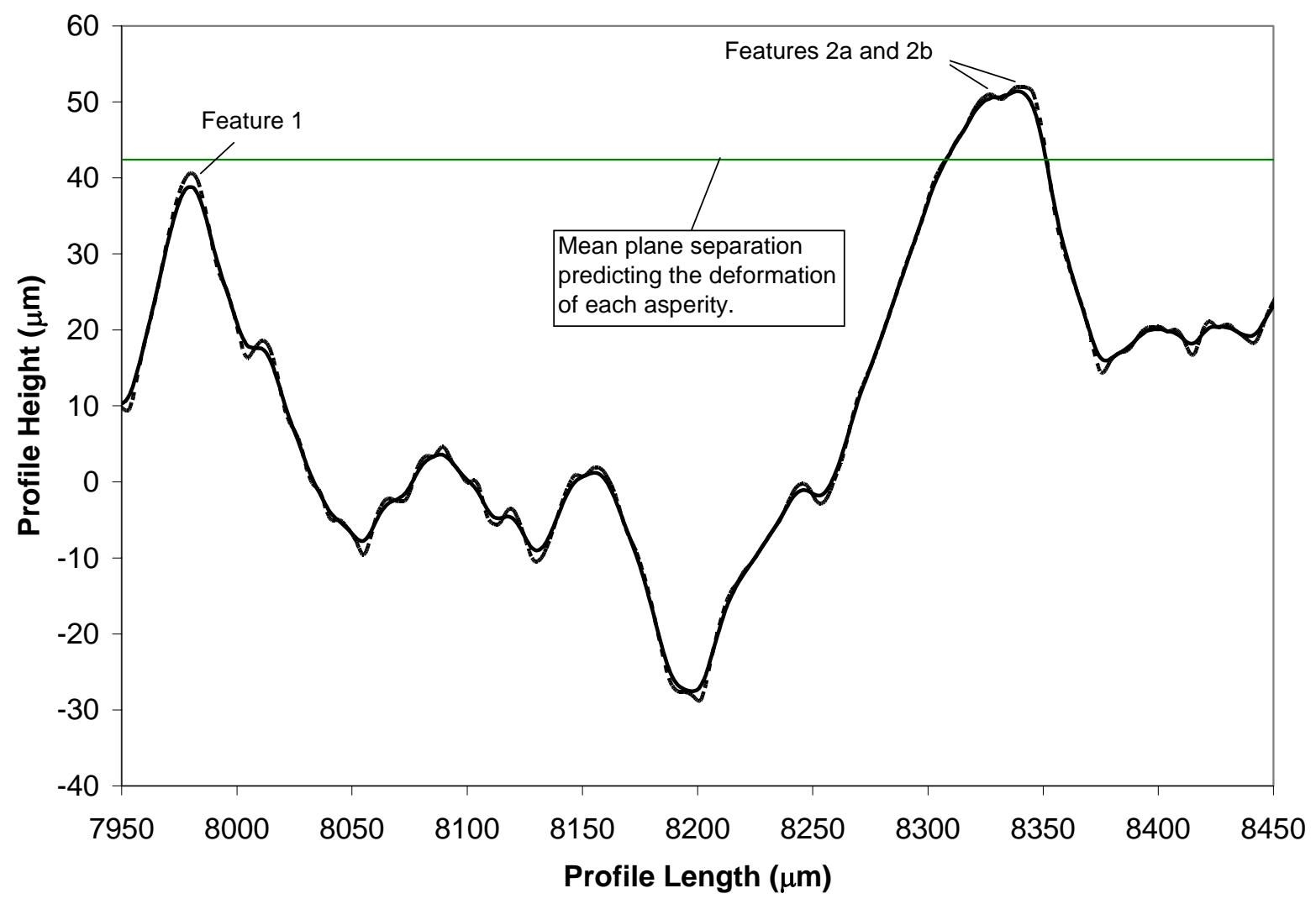

Figure 7: Unfiltered and filtered $10 \mu \mathrm{m}$ aluminum scan along with predicted deformation at $2600 \mathrm{~N}$ load. 


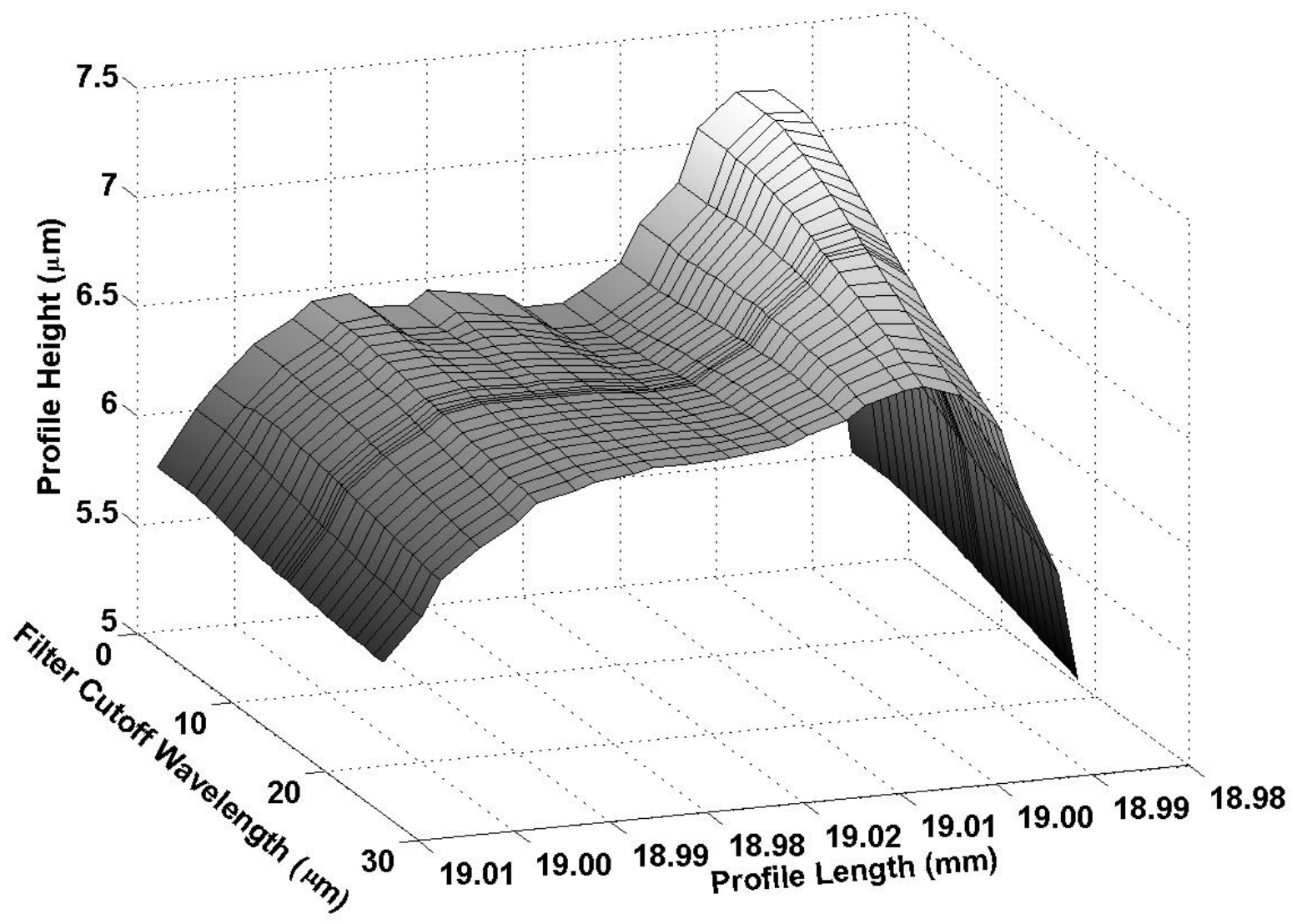

Figure 8: Effect of filtering on two small asperities as they merge into one large asperity. 


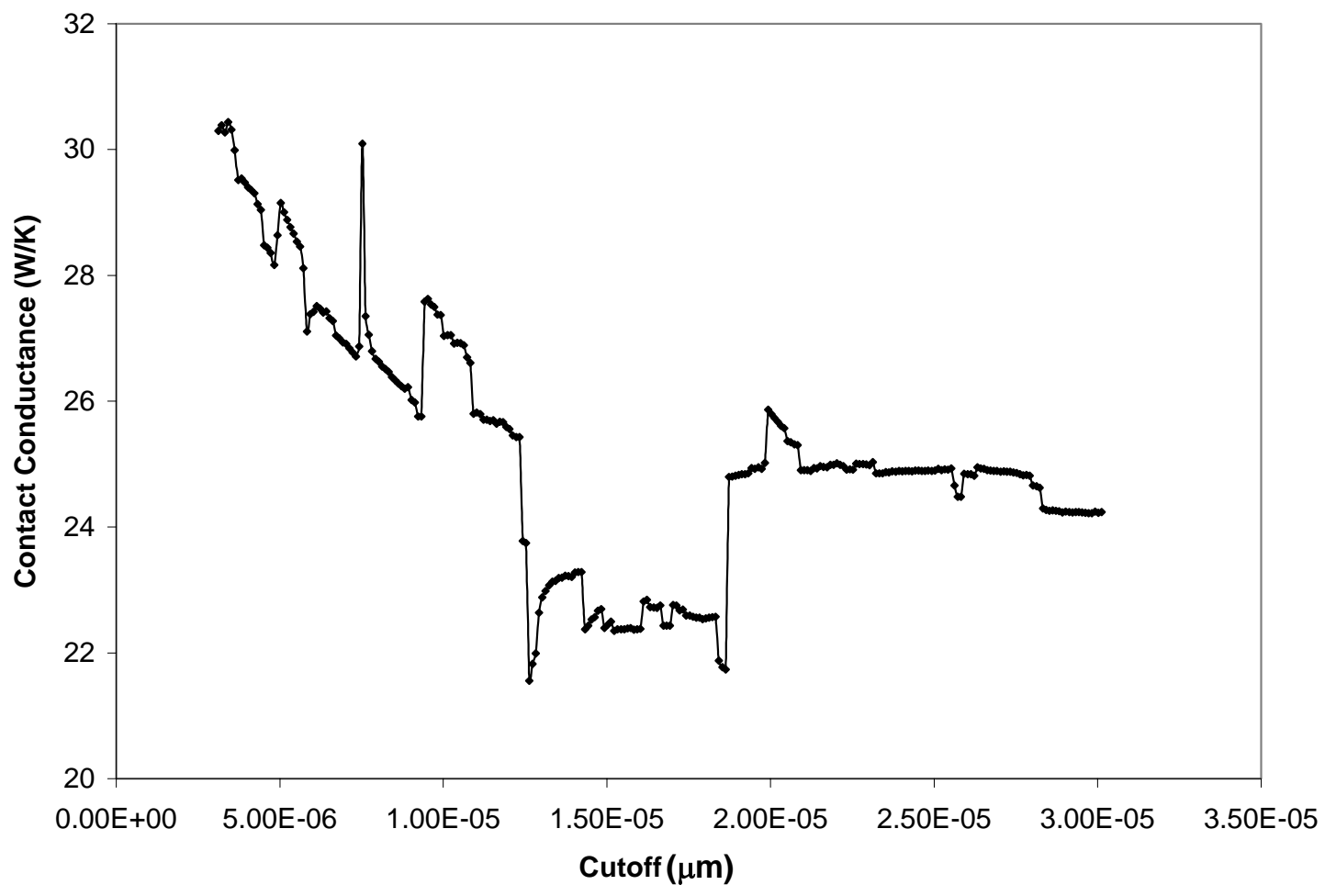

Figure 9: Predicted thermal contact conductance of a $1 \mu \mathrm{m}$ Ra aluminum surface at a load of $1550 \mathrm{~N}$ as a function of cutoff wavelength. 


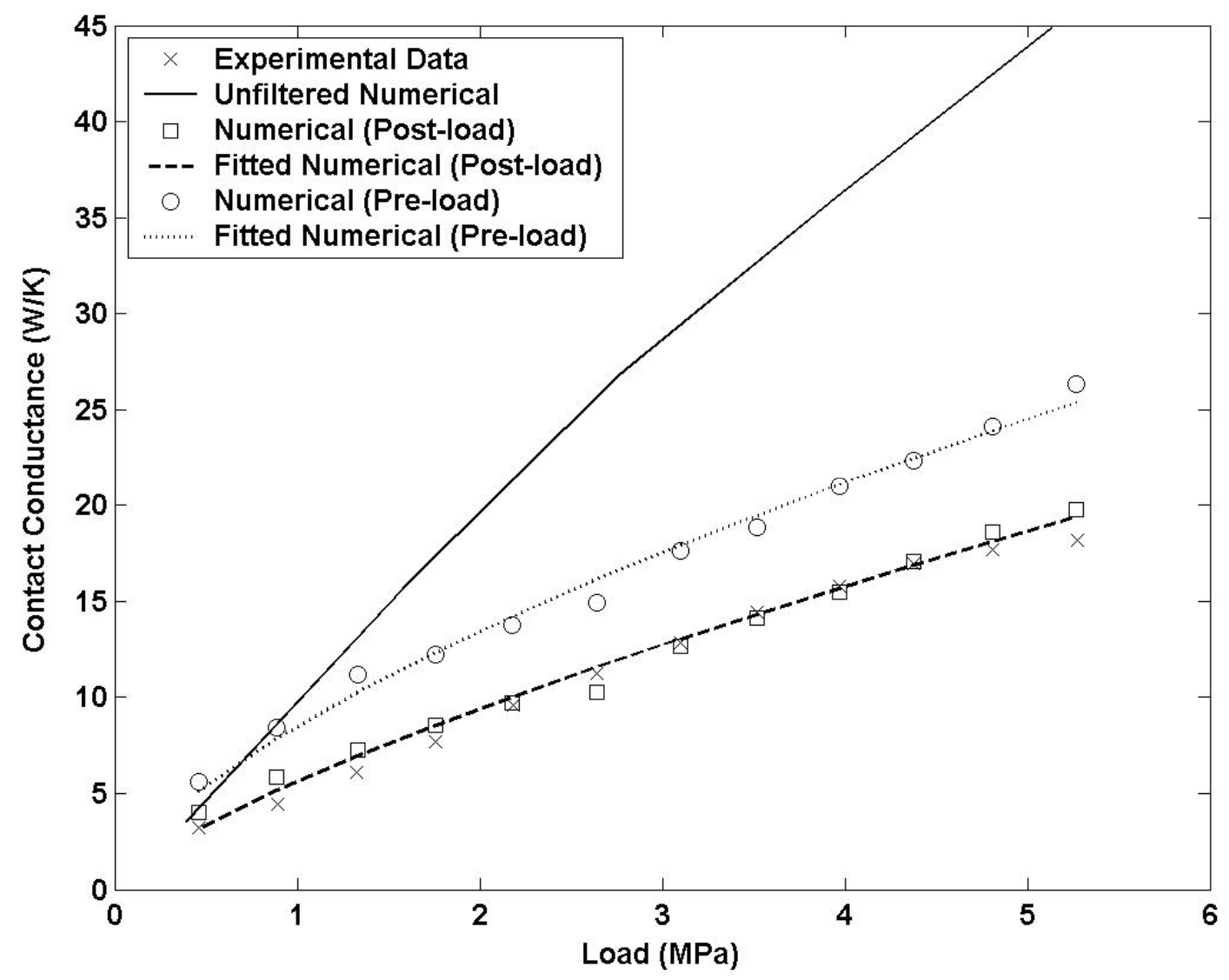

Figure 10: Comparison of experimental contact conductance versus load for a $1 \mu \mathrm{m}$ rough aluminum surface to numerically predicted values for filtered profile scans; predictions from unfiltered scans are also shown. 


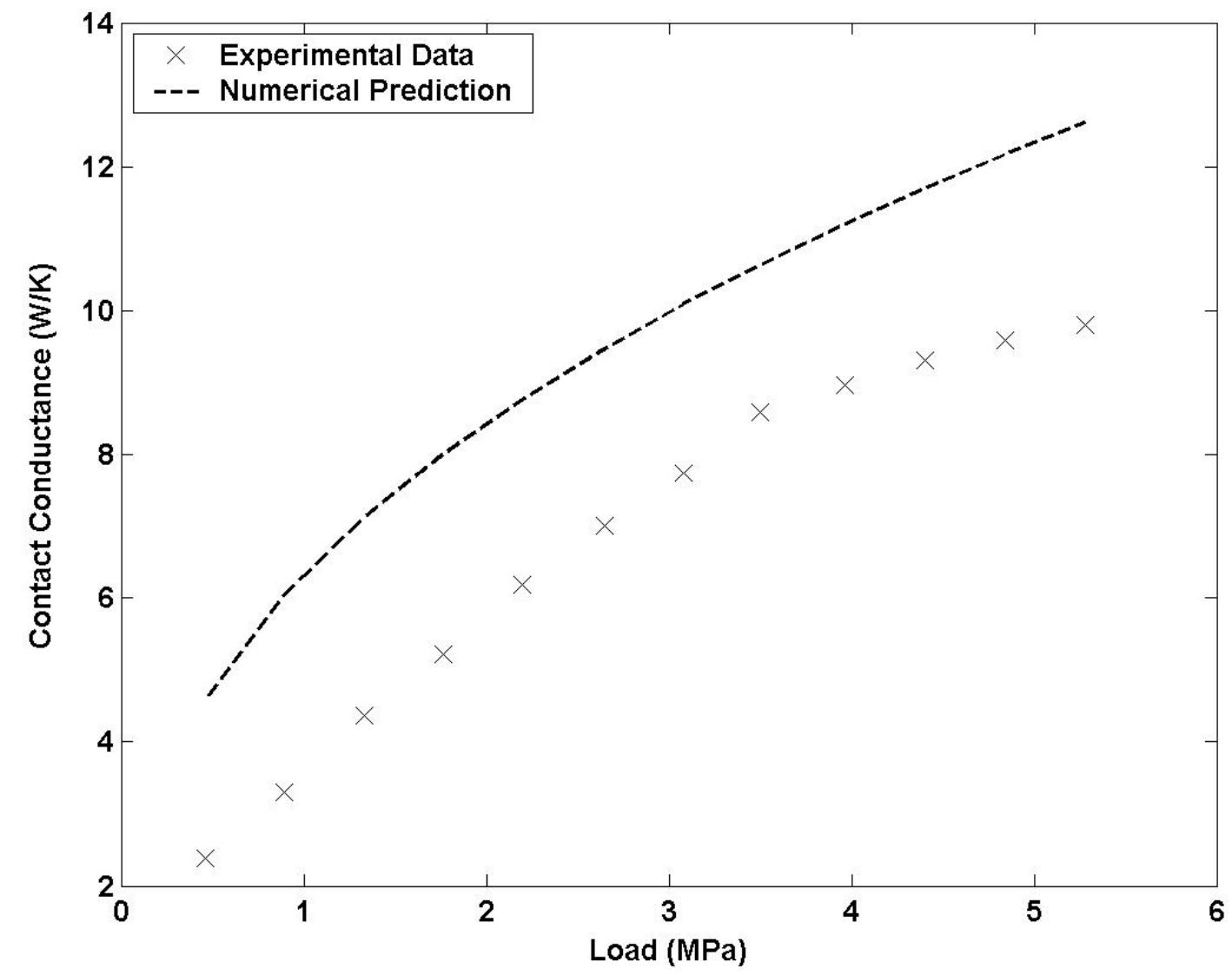

Figure 11: Comparison of experimental contact conductance versus load for a $5 \mu \mathrm{m}$ rough aluminum surface to predicted values from post-load scans. 


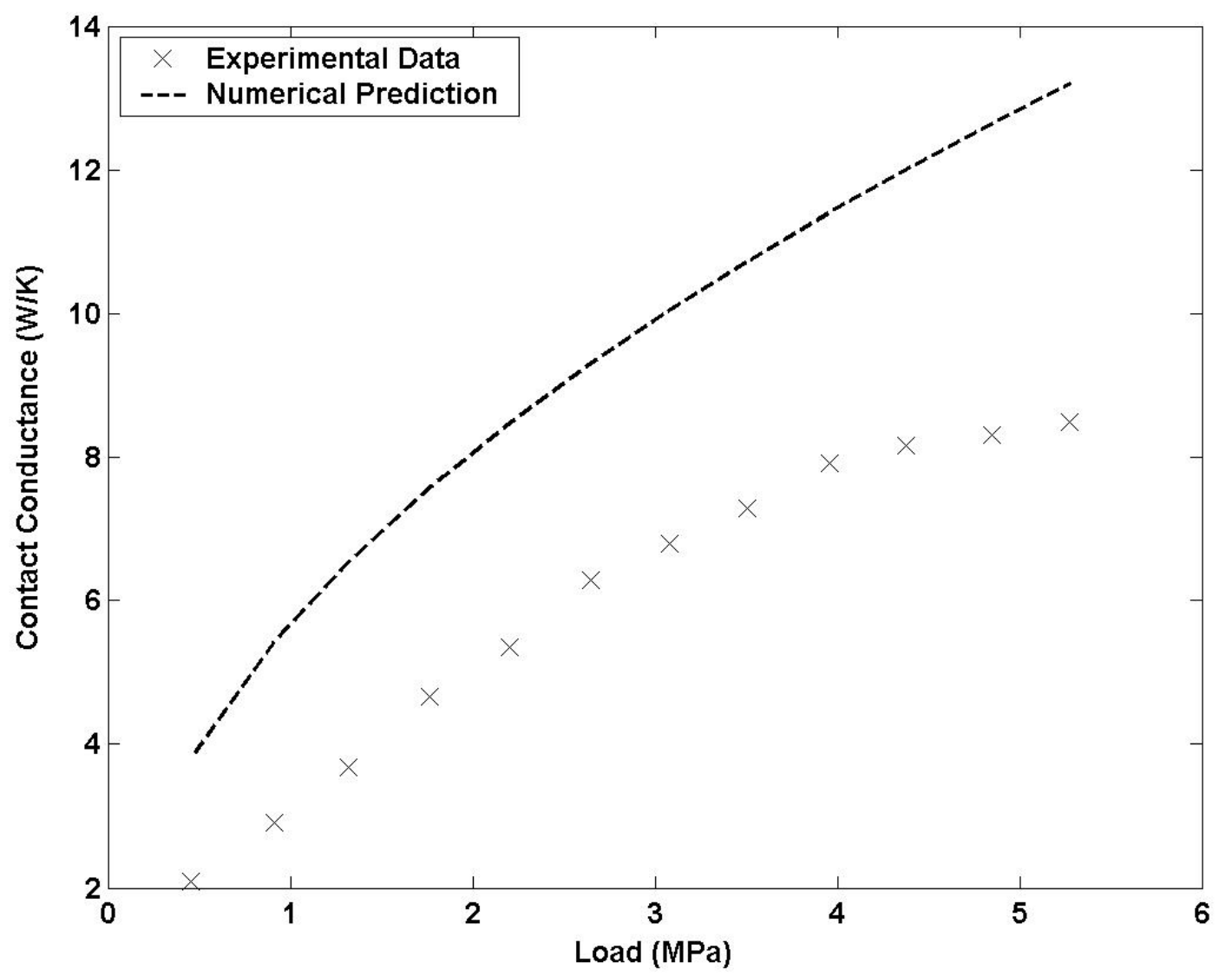

Figure 12: Comparison of experimental contact conductance versus load for a $10 \mu \mathrm{m}$ rough aluminum surface to predicted values from post-load scans. 


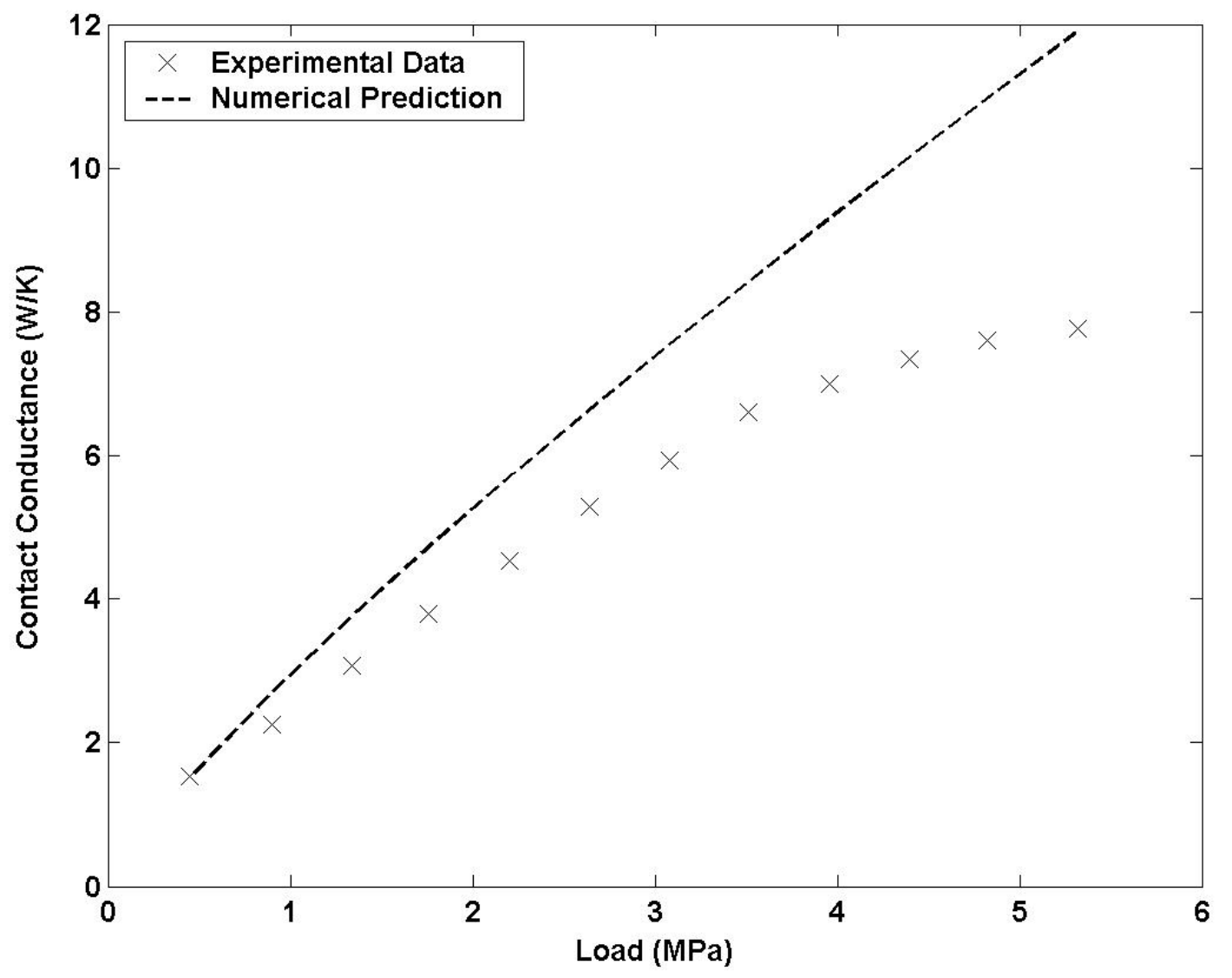

Figure 13: Comparison of experimental contact conductance versus load for a $15 \mu \mathrm{m}$ rough aluminum surface to predicted values from post-load scans. 


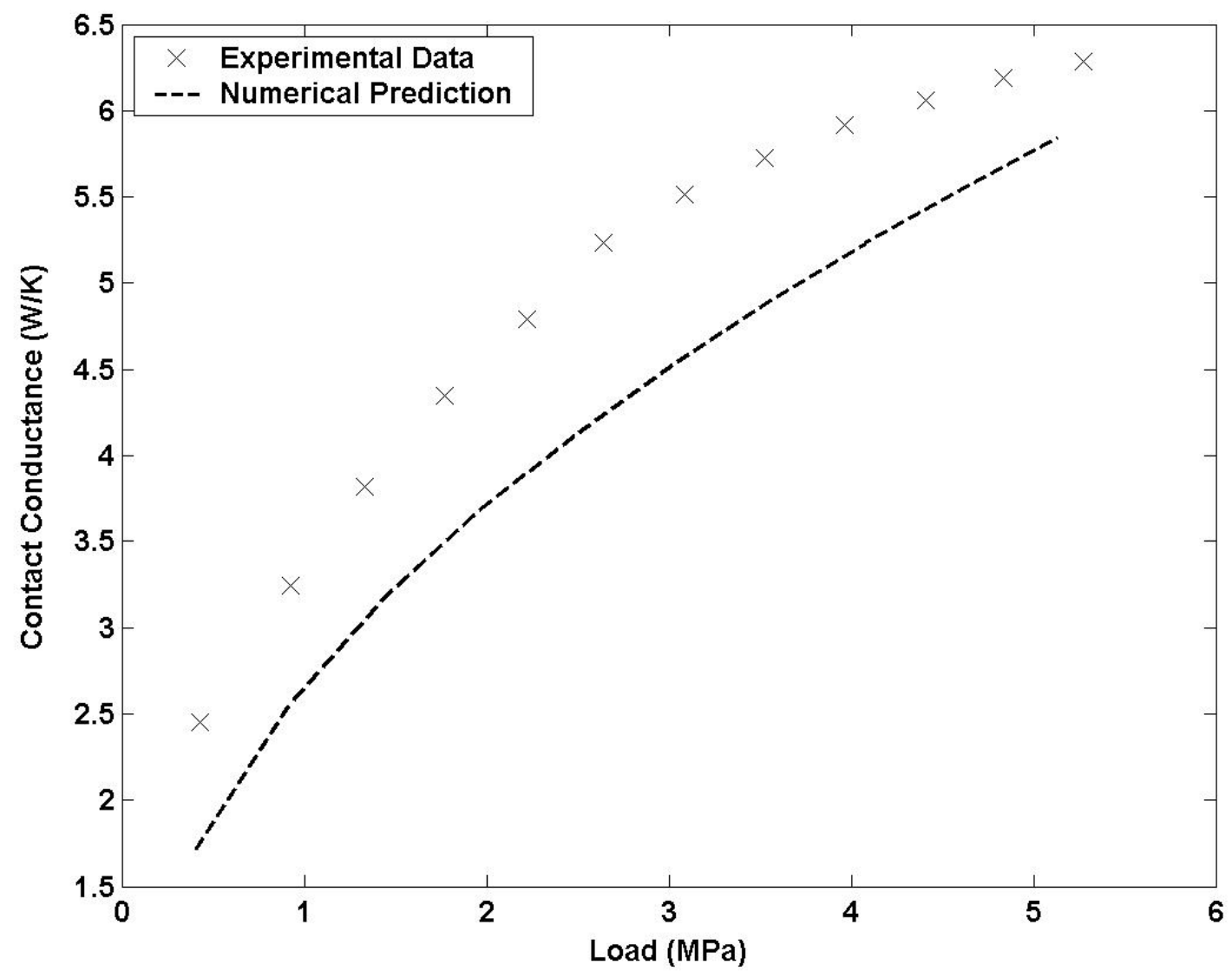

Figure 14: Comparison of experimental contact conductance versus load for a $5 \mu \mathrm{m}$ rough brass surface to predicted values from post-load scans. 


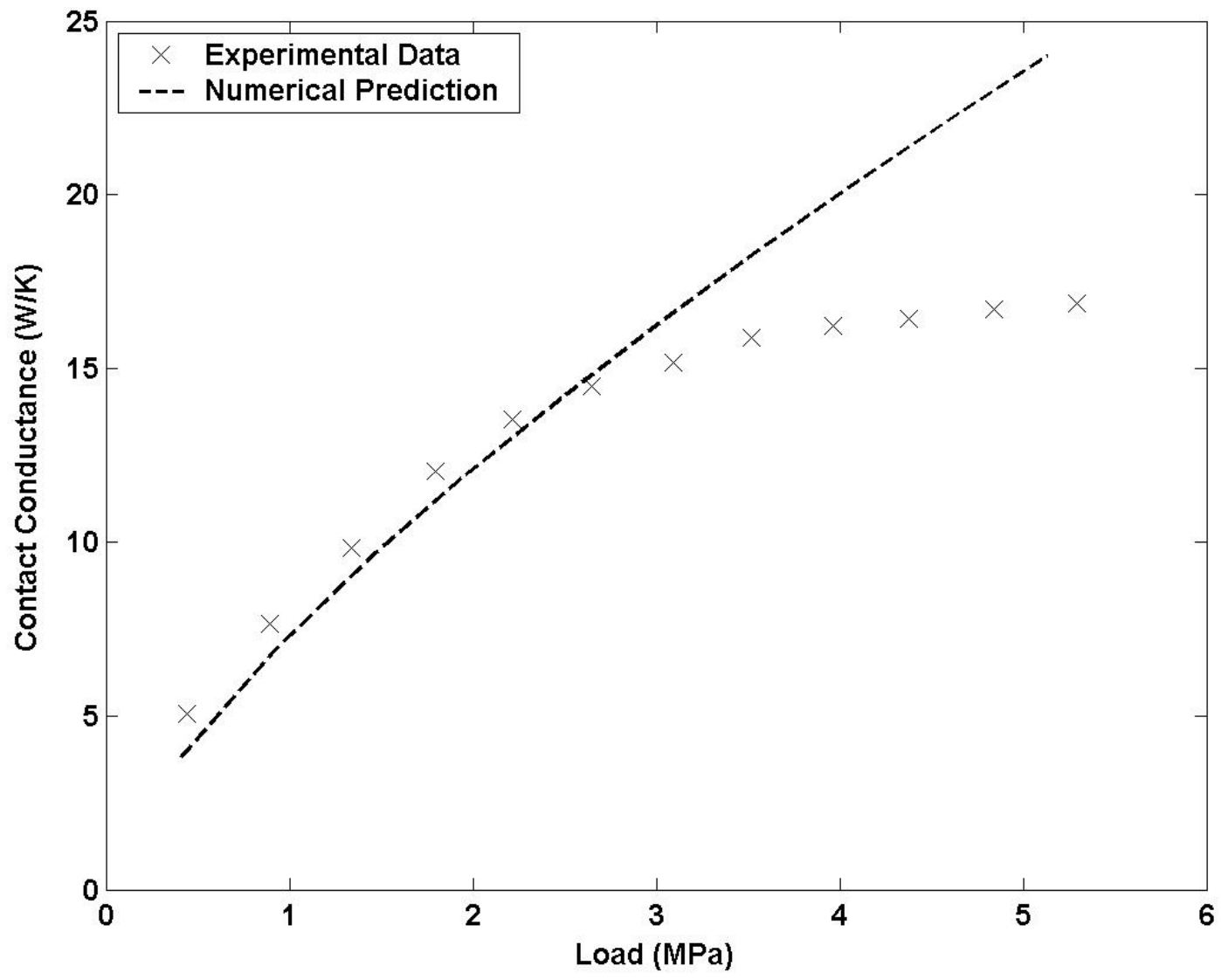

Figure 15: Comparison of experimental contact conductance versus load for a $5 \mu \mathrm{m}$ rough copper surface to predicted values from post-load scans. 